Draft Version November 5, 2018

Preprint typeset using $\mathrm{IAT}_{\mathrm{E}} \mathrm{X}$ style emulateapj v. 5/2/11

\title{
THE FORMATION OF YOUNG DENSE STAR CLUSTERS THROUGH MERGERS
}

\author{
M. S. FujiI ${ }^{1}$, T. R. SAitoh ${ }^{2}$, And S. F. Portegies Zwart ${ }^{1}$ \\ Draft version November 5, 2018
}

\begin{abstract}
Young star clusters like R136 in the Large Magellanic Cloud and NGC 3603, Westerlund 1, and 2 in the Milky Way are dynamically more evolved than expected based on their current relaxation times. In particular, the combination of a high degree of mass segregation, a relatively low central density, and the large number of massive runaway stars in their vicinity are hard to explain with the monolithic formation of these clusters. Young star clusters can achieve such a mature dynamical state if they formed through the mergers of a number of less massive clusters. The shorter relaxation times of less massive clusters cause them to dynamically evolve further by the time they merge, and the merger product preserves the memory of the dynamical evolution of its constituent clusters. With a series of $N$-body simulations, we study the dynamical evolution of single massive clusters and those that are assembled through merging smaller clusters together. We find that the formation of massive star clusters through the mergers of smaller clusters can reproduce the currently observed spatial distribution of massive stars, the density, and the characteristics (number and mass distribution) of the stars ejected as runaways from young dense clusters. We therefore conclude that these clusters and possibly other young massive star clusters formed through the mergers of smaller clusters.

Subject headings: methods: numerical - open clusters and associations: individual (R136, NGC 3603, Westerlund 1, Westerlund 2) - galaxies: star clusters
\end{abstract}

\section{INTRODUCTION}

Young ( $\lesssim 4 \mathrm{Myr})$ and dense $\left(\rho \gtrsim 10^{4} M_{\odot} / \mathrm{pc}^{3}\right)$ star clusters, NGC 3603, Westerlund 1 and 2 in the Milky Way Galaxy and R136 in the Large Magellanic Cloud (LMC), pose a number of interesting evolutionary problems on the formation and early evolution of star clusters. Their relatively small number of stars and their young ages make them ideal modeling targets (see Portegies Zwart et al. 2010), because they can be simulated on a star-by-star basis for their entire lifetime. In addition, the observational data on these clusters have excellent quality, which makes them ideally suited for comparison with the results of the numerical simulations.

From an observational point of view, each of these clusters shows a remarkably mature dynamical state compared to their ages (Haravama et al. 2008; Andersen et al. 2009; Ascenso et al. 2007; Gennaro et al. 2011); the degree of mass segregation suggests that either these clusters are born with a certain degree of mass segregation or that it develops more quickly than expected by standard relaxation theories.

In particular, the LMC cluster, R136, shows characteristics of a post-core-collapse star cluster (Mackey \& Gilmore 2003; Fuiii \& Portegies Zwart 2011, hereafter FPZ2011). The cuspy density profile of R136 (Mackey \& Gilmore 2003) and the large number of massive runaway stars which seem to have escape from R136, such as VFTS 682 (Bestenlehner et al. 2011), 30 Dor 016 (Evans et al. 2010) and several OB stars (Brandl et al. 2007; Gvaramadze et al. 2010), are effective signatures of core collapse. The mass and number distribution of

\footnotetext{
fujii@strw.leidenuniv.nl

${ }^{1}$ Leiden Observatory, Leiden University, NL-2300RA Leiden, The Netherlands

${ }^{2}$ Interactive Research Center of Science, Tokyo Institute of Technology, 2-12-1 Ookayama, Meguro, Tokyo 152-8551, Japan
}

the runaway stars ejected from R136 can be explained by three-body interactions between a hard binary and a single star (FPZ2011; Gvaramadze \& Gualandris 2011; Banerjee et al. 2012). Such a "bully" binary (BB) that formed in the gravothermal collapse of the cluster kicks out its surrounding stars and produces runaway stars. In FPZ2011, we demonstrated that the number of runaway stars that one BB can produce does not depend on the global parameters of the cluster, and accounts for $\sim 21$ in total. The fraction of runaway stars to the whole cluster, $f_{\text {run }}$, is therefore larger in clusters with fewer stars. The observed fraction of runaway stars is satisfactorily explained by the cluster having experienced core collapse within 1 Myr since its birth (see FPZ2011).

Such an early core collapse can be initiated sufficiently quickly if the initial density of the cluster core $\rho_{\mathrm{c}} \gtrsim 10^{6} M_{\odot} \mathrm{pc}^{-3}$, which is considerably higher than the currently density observed in R136: $\rho_{\mathrm{c}} \sim 5 \times 10^{4} M_{\odot} \mathrm{pc}^{-3}$ (Mackey \& Gilmore 2003). With a current half-mass relaxation time of $t_{\mathrm{rh}} \sim 100 \mathrm{Myr}$ (Mackey \& Gilmore 2003), it is unlikely that R136 evolved to a state of core collapse within $1 \mathrm{Myr}$ and subsequently evolved towards the less dense state it is in today.

One way to speed up the initial dynamical evolution towards a state of core collapse is by forming the cluster from the hierarchical merging of several smaller clusters (McMillan et al. 2007). The shorter relaxation timescales of these smaller clusters cause them to evolve dynamically more quickly than one single large cluster (Aarseth \& Hills 1972; McMillan et al. 2007; Moeckel \& Bonnell 2009; Yu et al. 2011). A merger between these dynamically evolved clusters preserves the memory of their past dynamical evolution and imprints this onto the more massive post-merger clusters (McMillan et al. 2007).

Sub-clustered star formation seems to be common 
for young stars and protostars embedded in molecular clouds (Gutermuth et al. 2009; Lada \& Lada 2003; Portegies Zwart et al. 2010). Numerical simulations of star formation in turbulent molecular clouds also support star cluster formation via hierarchical mergers (Bonnell et al. 2003, 2004, 2011; Girichidis et al. 2011; Kruijssen et al. 2012). Not only for young clusters in the Galactic disk, the merger scenario is suggested for extended old globular clusters in the Milky Way-like NGC2419 (Brüns \& Kroupa 2011; Brüns et al. 2011) and massive star clusters which are born during galaxy mergers (Saitoh et al. 2011). Thus, the formation of star clusters via hierarchical merging of sub-clusters might be very common for any kinds of star clusters.

The hierarchical merging of small sub-star clusters solves two problems in our understanding of R136 and other clusters with a similar age, density, mass, and mass function: (1) It allows for the cluster with a long relaxation time to experience core collapse well within the time frame for these sub-clusters, and (2) because each of the sub-clusters experiences an individual core collapse before they merge, the number of runaway stars produced by dynamical sling shots is considerably higher. Since the number of runaways is independent of cluster mass (FPZ2011), the expected number of O-type runaway stars is only one or two for R136 if it formed as a single cluster, but at least three were observed (Gvaramadze et al. 2010; Evans et al. 2010). It is therefore surprising that R136 has produced more runaways than anticipated by this theory if it was born as a single cluster. The hierarchical merging formation of R136 solves this conundrum.

The hierarchical merging of star clusters is also preferable for the formation of very massive $\left(>150 M_{\odot}\right)$ stars found in R136 and NGC 3603 (Crowther et al. 2010). Runaway collisions of stars during core collapse easily form such very massive stars in the cluster core (Portegies Zwart et al. 1999; Fujii et al. 2009). These massive stars stay in the cluster center due to mass segregation and form BBs. In this way, each sub-cluster that experiences core collapse will lead to the formation of one BB. After the sub-clusters merged, the massive BBs interact with each other in the core of the merged cluster. The binary-binary interactions can form a massive escaping binary like R145 (Schnurr et al. 2009; Gvaramadze \& Gualandris 2011).

We also argue that Westerlund 1 and 2 are still in the hierarchical merging process, which is demonstrated by their clumpy appearance. These clusters have not completed their merging processes, which has profound consequences for their current degree of mass segregation and the distribution of their stars (Mengel \& Tacconi-Garman 2009; Gennaro et al. 2011; Roman-Lopes et al. 2011).

In this paper, we demonstrate that merging star clusters can explain the dynamically evolved characteristics of young dense clusters in the Milky Way and the LMC. The observed distributions of massive stars including stars with an initial mass of $\gtrsim 150 M_{\odot}$, such as those found in R136 and NGC 3603, which are consistent with our results. Furthermore, the runaway stars around R136 and Westerlund 2 are consistent with our merger models. The core collapse in each small sub-cluster before merger forms a massive hard binary in its core. These massive binaries in the sub-clusters produce a sufficient amount of runaway stars, and then they are ionized or ejected from the cluster after their hosts merged. We demonstrate that such massive stars exhibit a spatial distribution similar to those observed in the region of R136 and NGC 3603. These dynamical processes result in a relatively low density of the cluster compared to their initial density or the density during the core collapse; the core density of these merger remnants in our simulations is consistent with those of observed young clusters.

\section{METHODS}

We performed a series of $N$-body simulations of single and multiple star clusters that merge to form a large single cluster. For merger cases, we adopted a mass of $6.3 \times 10^{3} M_{\odot}$ and a King model (King 1966) with the dimensionless central potential $W_{0}=2$ for each subcluster. The initial mass function (IMF) of stars were drawn from the Salpeter mass function (Salpeter 1955) between 1 and $100 M_{\odot}$. We imposed that each cluster contains at least one star with $m>80 M_{\odot}$. The initial half-mass radius and core density of our simulated clusters are $r_{\mathrm{h}} \simeq 0.1 \mathrm{pc}$ and $\rho_{\mathrm{c}} \simeq 2 \times 10^{6} M_{\odot}$ $\mathrm{pc}^{-3}$, respectively. This core density is similar to the central density of sub-clusters formed in star formation simulations in a turbulent molecular cloud (Bonnell et al. 2004). With these initial conditions our model clusters have $N=2048(2 \mathrm{k})$ stars and a half-mass relaxation time of $t_{\mathrm{rh}} \simeq 0.37 \mathrm{Myr}$. We call this model single- $2 \mathrm{k}$. The parameters are summarized in table 1.

We initially distribute 4 or 8 of these sub-clusters (single-2k) randomly in a volume with a radius of $r_{\max }$ and with zero velocity. We varied $r_{\max }$ from 1 pc to $6 \mathrm{pc}$. The clusters merge within a few Myr to a single cluster. The total masses of the merger remnant correspond to the mass of NGC 3603 for 4-cluster mergers and R136 for the 8-cluster merger cases. We summarize the initial conditions in table 2 .

For comparison, we also performed simulations of single clusters, whose masses are identical to R136. For this single cluster model, we adopted a King model for the initial density profile with $W_{0}=6$; we call this model single-w6. The core density of model single-w6 is comparable to those of the individual single- $2 \mathrm{k}$ models, but with a total mass $M \simeq 5 \times 10^{4} M_{\odot}$ and a half-mass radius $r_{\mathrm{h}} \simeq 0.3 \mathrm{pc}$. The resulting half-mass relaxation time, $t_{\mathrm{rh}}$, is $\sim 4.4 \mathrm{Myr}$, which enables these clusters to experience collapse within $\sim 1$ Myr (Portegies Zwart \& McMillan 2002; Gürkan et al. 2004).

The models, single-2k and single-w6, have a higher initial central density than those of observed star clusters. This choice of initial density is motivated by our requirement that the cluster should experience core collapse within $\sim 1$ Myr, which is required for the clusters to effectively to produce runaway stars. However, the core density drops and becomes comparable to the observed ones after the core collapse due to the scattering of stars in the cluster center. We discuss more details in section 3.1 .

We additionally performed simulations of a single model with the same parameters as single-w6 but with a smaller lower-mass limit of the IMF. For our standard models, we adopted $1 M_{\odot}$ as a lower-mass limit, which is more massive than observed ones. We adopted $0.47 M_{\odot}$ 
as a lower-mass limit for this model (single-lm), and as a consequence the mean mass of single-lm is half of singlew6.

Furthermore, we performed two additional series of calculations of single clusters with the same mass and IMF as for the single-w6 model. One of these models has $W_{0}=8$ and half-mass radius $r_{\mathrm{h}} \simeq 1.4 \mathrm{pc}$; we call this model single-w8. This model initially has a density profile similar to R136. The core density for this model is $1.6 \times 10^{5} M_{\odot} \mathrm{pc}^{-3}$ and its relaxation time is $\sim 43 \mathrm{Myr}$; this cluster is therefore not expected to experience core collapse within $~ 3 \mathrm{Myr}$ (Portegies Zwart \& McMillan 2002; Gürkan et al. 2004). The other model is with primordial binaries; which we name single-pb. We assigned the most massive $3 \%$ of stars $\left(\gtrsim 8 M_{\odot}\right)$ as equal-mass primordial binaries with the binding energy of $3 \mathrm{kT}$, which corresponds to an orbital period of $\sim 3 \times 10^{3}$ $10^{6}$ days. Here $\mathrm{kT}$ is the fundamental unit of kinetic energy and $1.5 \mathrm{kT}$ corresponds to the average kinetic energy of stars in the cluster before we add binaries. Binaries with this binding energy have intermediate hardness and therefore interact efficiently with single stars and with each other (Tanikawa \& Fukushige 2009) and therefore they are expected to produce runaway stars, while binaries with shorter orbital periods are dynamically inactive due to their small cross sections (FPZ2011). We choose equal-mass binaries because massive stars observationally prefer massive companions (Sana \& Evans 2011; Zinnecker \& Yorke 2007). All binaries are initialized with circular orbits and random orbital phase and inclination. In table 2 we summarize the initial conditions for these models.

During our simulations, we applied mass loss due to stellar winds only for stars more than $100 M_{\odot}$. Initially our simulations do not contain such massive stars, but they form occasionally due to collisions, for which we adopted the sticky-sphere approach. For the rate of mass loss by the stellar wind, for stars with $m>100 M_{\odot}$, we adopted $\dot{m}=5.7 \times 10^{-8}\left(\mathrm{~m} / M_{\odot}\right)\left(M_{\odot} \mathrm{yr}^{-1}\right)$ (Fuiii et al. 2009). The stellar radii were adopted to have zero-age main-sequence radii for solar metalicity (Hurley et al. 2000).

Each simulation was performed up to an age of $3 \mathrm{Myr}$, which is the time for the first supernova explosion. The equations of motion were integrated using a sixth-order Hermite scheme with individual timesteps with an accuracy parameter $\eta=0.15-0.3$ (Nitadori \& Makino 2008). We adopted the accuracy parameter to balance speed and accuracy. Our code does not include any special treatment for hard binaries. The sixth-order Hermite scheme, however, can deal with hard binaries which form in our simulations. We tested our code integrating hard binaries with an eccentricity of $>0.9$. Figure 1 shows an orbital separation of a binary. The masses of the binary components are 100 and $150 M_{\odot}$, respectively, and their orbital period, semi-major axis, and eccentricity are 72 year, $109 \mathrm{AU}$, and 0.96. This binary has the same parameters as one of hard binaries which actually formed in our simulations. The energy and angular momentum errors maintain $<10^{-6}$ and $<2 \times 10^{-8}$ in 0.1 Myr. Even if the energy error increases linearly, the relative error in the energy is conserved to less than $0.1 \%$ over the $3 \mathrm{Myr}$ of the simulation: our code treats close stellar encounters down to $\sim 1$ AU accurately. Stars that approach each other within 1 AU generally participate in a collision and therefore such close encounters do not need to be resolved. For each simulation we confirmed that the energy error of clusters is below $0.1 \%$ of the initial total energy of the clusters.

\section{COMPARISON BETWEEN SIMULATIONS AND OBSERVATIONS}

After $3 \mathrm{Myr}$, the sub-clusters merged to one cluster for all merger simulations. We present the time evolution of model m2k8r6 until $3 \mathrm{Myr}$ in Figure 2. The subclusters hierarchically merge and finally form one star cluster with a number of escaping stars. The merger remnants are almost spherical and look very similar to initially spherical single clusters. However, the distribution of stars in the merged clusters are different from that of clusters that were formed as one. In this section we demonstrate that the merger scenario is preferable for the formation of young dense clusters.

\subsection{Cumulative number of massive stars}

The projected cumulative distributions of massive stars as a function of the distance from the cluster center at a cluster age of $3 \mathrm{Myr}$ are presented in Figure 3. We adopted the density center (Casertano \& Hut 1985) as the cluster center. The black dashed and dotted curves show the results for the single clusters, whereas the colored curves show the results for the merger simulations. We plot massive stars with $>30 M_{\odot}$ for the simulations. For comparison, we overlay the observed distribution for massive stars Wolf-Rayet (WR), spectral type O, and some early B stars from R136 (left) and from NGC 3603 (right) Crowther \& Dessart 1998, thick black curve with crosses). Although the mass range we adopted for the simulations is higher than that of the observation, the fraction of plotted stars to the total mass of the cluster is similar for both the simulation and the observation. If we assume that a Salpeter mass function between 0.3-100 $M_{\odot}$, the fraction of O-stars $\left(>16 M_{\odot}\right)$ is $13.5 \%$, which corresponds to the fraction of stars with $>30 M_{\odot}$ assuming a Salpeter mass function between $1-100 M_{\odot}$.

In the left panel of Figure 3, we find that the merger models and the single cluster with a relatively low central density (single-w8) are consistent with R136. We quantify the spatial distribution of the massive stars using the radius of the inner tenth star, $r_{10}$. These values are $0.11 \pm 0.02,0.087$, and $0.15 \mathrm{pc}$ for merger models, single-w8, and R136, respectively. Model single-w8 does not experience core collapse sufficiently quickly to explain the high degree of mass segregation inside the star cluster, nor does this model produce the observed number of runaway stars (the detail is in section 3.2). On the other hand, single models with a high central density (single-w6) and with primordial binaries (single-pb) can produce enough runaway stars, but produce too a concentrated distributions of massive stars. Their values of $r_{10}$ are $0.034 \pm 0.005$ and $0.025 \mathrm{pc}$ for single-w6 and single-pb, respectively, and these values are much smaller than that of R136. We also find the agreement between merger models and observation for NGC 3603 (see right panel of Figure 3).

We also see in the left panel of Figure 3 that a wider initial distribution of sub-clusters results in a relatively 
TABLE 1

Single CLUSTER MODELS.

\begin{tabular}{cccccccccc}
\hline Model & $N$ & $M\left(M_{\odot}\right)$ & $W_{0}$ & $r_{\mathrm{h}}(\mathrm{pc})$ & $\rho_{\mathrm{c}}\left(M_{\odot} \mathrm{pc}^{-3}\right)$ & $\sigma(\mathrm{km} / \mathrm{s})$ & $t_{\mathrm{rh}}(\mathrm{Myr})$ & $m_{\min }\left(M_{\odot}\right)$ & $N_{\mathrm{run}}$ \\
\hline \hline single-2k & 2048 & $6.3 \times 10^{3}$ & 2 & 0.097 & $2.2 \times 10^{6}$ & 11 & 0.37 & 1.0 \\
single-w6 & 16384 & $5.1 \times 10^{4}$ & 6 & 0.32 & $1.7 \times 10^{6}$ & 17 & 4.4 & 1.0 \\
single-lm & 32768 & $5.1 \times 10^{4}$ & 6 & 0.32 & $1.7 \times 10^{6}$ & 17 & 8.3 & 0.47 & 3 \\
single-w8 & 16384 & $5.1 \times 10^{4}$ & 8 & 1.4 & $1.6 \times 10^{5}$ & 6 & 43 & 1.0 \\
single-pb & 16875 & $5.1 \times 10^{4}$ & 6 & 0.32 & $1.7 \times 10^{6}$ & 17 & 4.4 & 1.0 \\
\hline
\end{tabular}

TABLE 2

MERGER MODELS.

\begin{tabular}{cccc}
\hline Model & $N_{\mathrm{cl}}{ }^{\mathrm{a}}$ & $r_{\max }(\mathrm{pc})^{\mathrm{b}}$ & $N_{\text {run }}{ }^{\mathrm{c}}$ \\
\hline \hline m2k8r1 & 8 & 1 & 1 \\
m2k8r3 & 8 & 3 & 1 \\
m2k8r5 & 8 & 5 & 2 \\
m2k8r6 & 8 & 6 & 2 \\
m2k4r3 & 4 & 3 & 3 \\
\hline
\end{tabular}

Note. - The models are named according to the following rules; "m" indicates merger models, the name of models for the sub-clusters, the number of sub-clusters, and the value of $r_{\max }$. For example, m2k8r1 stands for a merger model of eight single-2k clusters which are located within $r_{\max }=1 \mathrm{pc}$.

athe number of sub-clusters.

b The radius in which sub-clusters initially distribute.

${ }^{\mathrm{c}}$ The number of runs which we performed.
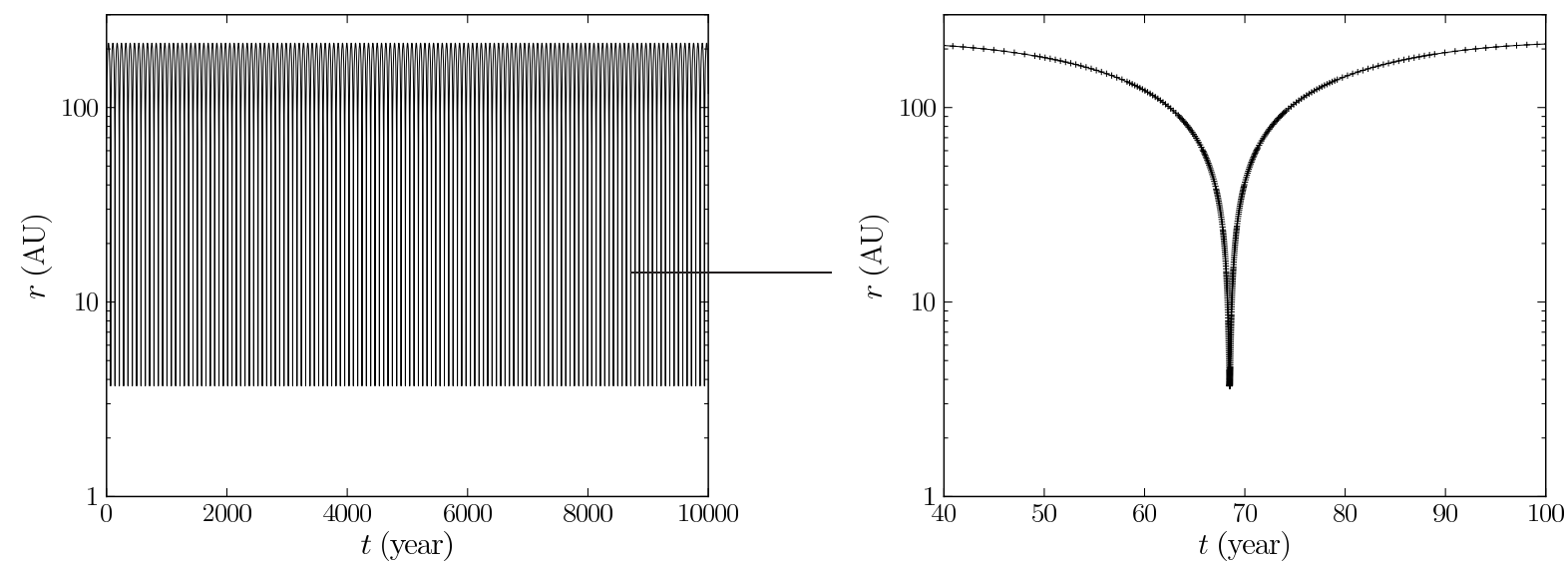

FIG. 1. - Orbital separation of a hard binary as a function of time (right for 0.1 Myr and left: for one orbital period). The masses of the binary components are 100 and $150 M_{\odot}$, respectively. The orbital period, semi-major axis, and eccentricity are 72 year, 109 AU, and 0.966, respectively. In right panel, crosses indicate the time steps adopted by our timestep criterion (Nitadori \& Makino 2008) with an accuracy parameter of 0.15 . The minimum and maximum stepsizes in this period are 0.73 day and 1.0 year, respectively.

wider distribution of massive stars after the mergers, i.e., the value of $r_{10}$ is larger with the larger $r_{\max }$. While $r_{10}=0.096 \pm 0.002 \mathrm{pc}$ for the models with $r_{\max }=1-3$ $\mathrm{pc}, r_{10}=0.12 \pm 0.03 \mathrm{pc}$ for the models with $r_{\max }=5-6$ pc. The wider models can reproduce the distribution of massive stars in R136 better.

We find that the degree of dynamical evolution in each of the sub-clusters before they merge makes this difference. The sub-clusters merge after they experience core collapse for the majority of our simulations except model $\mathrm{m} 2 \mathrm{k} 8 \mathrm{r} 1$. In the latter case, the time scales for merger and core collapse are comparable. One would naively expect that the more dynamically developed sub-clusters will cause the merger product to be more centrally concentrated also, but that is only partially true. The dynami- cally evolved sub-clusters results in the lower concentration because of their shorter relaxation time. Figure 4 shows the time evolution of the core density for single clusters. The isolated sub-clusters (single-2k) collapse at $\sim 0.3 \mathrm{Myr}$ and then the core density decreases dramatically due to the scattering of stars by hard binaries formed in a sub-clusters before they merged. This lower core density after the core collapse induces the lower concentration of the merger remnants. Contrary to this, initially massive single clusters maintain their high densities much longer.

The wider initial distribution of sub-clusters provides sufficient time for them to experience core collapse and produce a BB, which ejects massive stars from each subcluster (FPZ2011). The post-collapse sub-clusters have 
a lower density than those in a state of core collapse. After the sub-clusters merge, however, most of the BBs are broken or scattered up due to binary-binary interactions (Gualandris et al. 2004), and the production of massive runaways is finally driven by one single BB. The remaining cluster starts to evolve as an ordinary single cluster. This explains the wider radial profile of the more massive stars. We schematically represent this process in Figure 5

In Figure 6] we present the density profile of the simulated clusters at $3 \mathrm{Myr}$. The central density of the merger products has already been depleted except model m2k8r1. These merger models and the single model single-w8 show similar distributions of massive stars and density profiles. Only the most concentrated merger model, m2k8r1, has parameters similar to a single dense model, single-w6. The central density $(r \lesssim 0.2 \mathrm{pc})$ of the merger cases with $r_{\max }=5-6 \mathrm{pc}$ is similar to the observed central density of R136, 3-5 $\times 10^{4} M_{\odot} \mathrm{pc}^{-3}$ (Mackey \& Gilmore 2003), and the central density of the four-merger cases is comparable to the core density of NGC $3603, \geq 6 \times 10^{4} M_{\odot} \mathrm{pc}^{-3}$ (Haravama et al. 2008).

From the too concentrated distribution of massive stars, we can exclude the single high density models (single-w6 and single-pb) as viable models of R136. If we see only the radial distribution in the cluster, however, the difference between the merger and single cases is not clear.

\subsection{Mass distribution of runaway stars}

Another way to evaluate the degree of the dynamical evolution of young star clusters is finding runaway stars around the cluster. We demonstrated in FPZ2011 that the fraction of runaway stars around R136 and also their mass distribution are consistent with those scattered by a binary in the core of the host cluster.

In FPZ2011, we discussed how a single cluster can explain the fraction of runaway stars around R136. The degree of mass segregation in our model single-w6 is too strong compared with the observed distribution of massive stars (see Figure 3). On the other hand, if we assume a single cluster with a distribution of massive stars (single-w8) similar to R136, no runaway stars are produced because the relaxation time of this model $\left(t_{\mathrm{rh}}=44\right.$ $\mathrm{Myr}$ ) is too long to experience core collapse within $3 \mathrm{Myr}$ (see Figure 4).

We conclude that both the observed distributions of massive stars and runaway stars of R136 can be reproduced if the star cluster formed through the hierarchical merger of $\sim 8$ small sub-clusters. In Figure 7 we show that $f_{\text {run }}$, the fraction of runaway stars that escape their host cluster with $>30 \mathrm{~km} / \mathrm{s}$, agrees well with the WR and spectral type O- and early B-stars observed around R136 (square). The observed $f_{\text {run }}$ of early Btype stars $\left(8-16 M_{\odot}\right)$ is a few times smaller than that expected from our simulations. Assuming that the total mass of $6.0 \times 10^{4} M_{\odot}($ Portegies Zwart et al. 2010) with a Salpeter mass function between 1 and $100 M_{\odot}$ and that the runaway fraction obtained from our 8-merger models, we predict that there are $7 \pm 2$ early-B runaways still in the vicinity of $\mathrm{R} 136$. We also expect that $\sim 6$ late- $\mathrm{O}$ $\left(16-32 M_{\odot}\right)$ and $\sim 11$ early-O/WR $\left(>32 M_{\odot}\right)$ runaway stars exist around R136.

The lower-mass limit of the IMF does not affect on the fraction of runaway stars (see the right panel of Figure 7 ) because the three-body scattering by a binary in the cluster core is driven by massive stars gathering in the cluster core due to the mass segregation. Even if we change the lower-mass limit of the IMF, low-mass stars do not interact much frequently with the binary in the core. In Figure 8 we show the kinetic energy of runaway stars as a cumulative function of mass, which corresponds to the energy extracted from the binary by three-body scatterings. The contribution from low-mass stars is much smaller than that from massive stars. The core-collapse time is not sensitive to the lower-mass limit either, although the number of particles increases with a smaller lower-mass limit. From the results of $N$-body simulations, the core-collapse time, $t_{\mathrm{cc}}$, follows an equation such as $t_{\mathrm{cc}} \propto\left(m_{\max } /\langle m\rangle\right)^{-1.3} t_{\mathrm{rh}}$ (Gürkan et al. 2004), where $m_{\max }$ and $\langle m\rangle$ are lower-mass limit and mean mass, respectively. Since $\langle m\rangle \propto 1 / N$ and the relaxation time is roughly proportional to $N$, the core-collapse time depends on $N^{-0.3}$. The lower-mass limit of the IMF, however, changes the mass where the fraction of runaway stars starts increase because only stars which are more massive than this limit mass can sink to the cluster center (Fuiii et al. 2008). A smaller value of the lower-mass limit decrease this mass (see Figure 7 and Baneriee et al. 2012, in which they assumed an IMF between 0.3 and 150 $M_{\odot}$ and obtained very similar results to ours.). The limit mass is 13 and $9 M_{\odot}$ for our models with a lower-mass limit of 1 and $0.47 M_{\odot}$, respectively.

The consistency between the observed number of runaways and the density profile for R136 gives us confidence in our prediction of a relative fraction of runaway stars around NGC 3603 and other clusters. As we discussed before, the fraction of runaway stars does not depends on the lower-mass limit of the IMF. Therefore, we adopted both 0.3 and $1 M_{\odot}$ as the lower-mass limit and assumed the fraction of runaway stars obtained from our simulations a the lower-mass limit of $1 M_{\odot}$. Since the number of massive stars assuming a lower-mass limit of $0.3 M_{\odot}$ is $\sim 60 \%$ of that in the case of $1 M_{\odot}$, the expected number of runaway stars also decrease $\sim 60 \%$. Adopting a Salpeter mass function between $1 M_{\odot}$ and $100 M_{\odot}$ and a total cluster mass of $\sim 1.7 \times 10^{4} M_{\odot}($ (Rochau et al. 2010$)$, we predict that $\sim 7 \mathrm{O} / \mathrm{WR}$ stars $\left(>16 M_{\odot}\right)$ have been ejected from NGC 3603. If we assume a lower-mass limit of $0.3 M_{\odot}$, we predict $\sim 4 \mathrm{O} / \mathrm{WR}$ runaway stars. We summarize the expected number of $\mathrm{OB}$ runaway stars around young star clusters in table 3. We assumed 8merger models for R136 and Westerlund 1 and 4-merger models for NGC 3603 and Westerlund 2.

\subsection{Single cluster with primordial binaries}

Since the binary fraction of massive stars in star clusters is very high (20-80\%) (Zinnecker \& Yorke 2007, and references therein), and binary-binary interactions are common and can effectively produce runaways (Leonard \& Duncan 1988; Clarke \& Pringle 1992; Gualandris et al. (2004), a fraction of primordial binaries and their distribution in binding energies are expected to affect the fraction of runaway stars. We performed a simulation for a single cluster model with primordial binaries (single-pb) in order to quantify the effect of binaries. The radial distribution of massive stars (Figure 3) and the density profile (Figure 6) are very similar to 

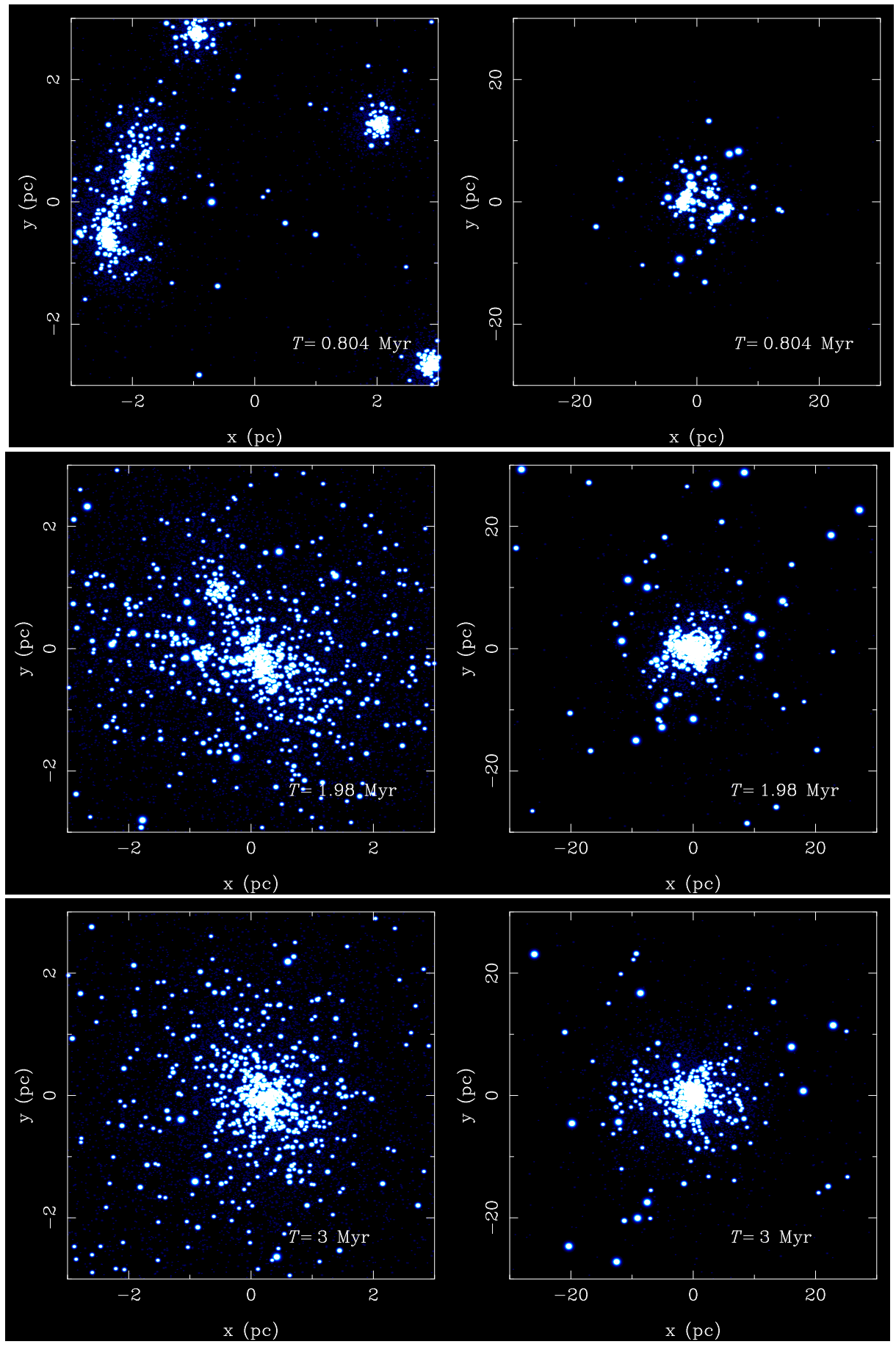

FIG. 2.- Snapshots of model m2k8r6-1 at 0.8, 2, and 3 Myr. Left column is the zoom-in of the right column.

the single case with the same initial density distribution (single-w6). The primordial binaries do not solve the problems that clusters require too high a core density and too concentrated a distribution of massive stars to form a sufficient amount of runaway stars; primordial binaries therefore do not help in reproducing the spatial distribution of the stars in R136.

The density profile of the cluster is not affected by the presence of primordial binaries, but the fraction of the runaway stars is higher compared to those produced in single clusters, and similar to those produced in merged cluster (see Figure 7). This fraction is consistent with the simulations performed by Baneriee et al. (2012).

\subsection{Comparison with Westerlund 1}

Westerlund 1 is another young massive star cluster in the Milky Way with a mass comparable to R136, but with an age of 3-6 Myr (Clark et al. 2005; Gennaro et al. 2011). The relaxation time estimated from its current mass and size is $\sim 130$ Myr, but the cluster is masssegregated (Gennaro et al. 2011). Furthermore, Wester- 

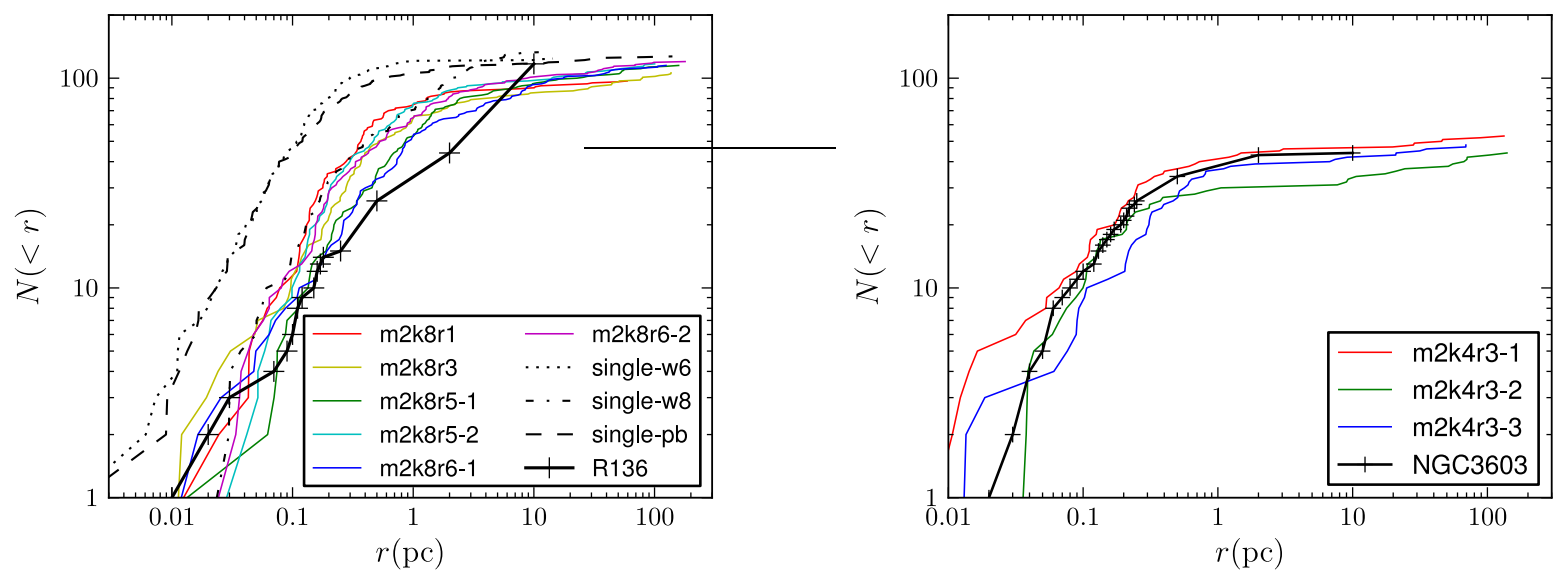

FIG. 3.- Projected cumulative number distribution of the massive ( $>30 M_{\odot}$ for simulations) stars for R136 (left) and NGC 3603 (right) models. Colored curves show merger models and black dashed, dash-dotted, and dotted curves show single models. Black crosses show the observation (Crowther \& Dessart 1998).

TABLE 3

EXPECTED RUNAWAY STARS.

\begin{tabular}{cccccccc}
\hline Name & $M_{\mathrm{cl}}\left(M_{\odot}\right)$ & Ref. & Model & $m_{\min }\left(M_{\odot}\right)$ & early-B & late-O & early-O/WR \\
\hline \hline R136 & $6.0 \times 10^{4}$ & 1 & 8-merger & 1 & $6.7 \pm 2.4$ & $6.4 \pm 3.0$ & $11 \pm 6$ \\
& & & & 0.3 & $4.9 \pm 1.5$ & $3.9 \pm 1.8$ & $6.5 \pm 3.5$ \\
NGC 3603 & \multirow{2}{*}{$1.7 \times 10^{4}$} & 2 & 4-merger & 1 & $2.9 \pm 2.2$ & $2.1 \pm 0.6$ & $4.8 \pm 1.5$ \\
& & & & 0.3 & $1.8 \pm 1.3$ & $1.3 \pm 0.3$ & $2.9 \pm 0.9$ \\
Westerlund 1 & \multirow{2}{*}{$6.3 \times 10^{4}$} & \multirow{2}{*}{1} & 8-merger & 1 & $7.0 \pm 2.5$ & $6.8 \pm 3.2$ & $11 \pm 6$ \\
& & & & 0.3 & $4.2 \pm 1.5$ & $4.1 \pm 1.8$ & $6.8 \pm 3.7$ \\
Westerlund 2 & \multirow{2}{*}{$10^{4}$} & \multirow{2}{*}{ 4-merger } & 1 & $1.7 \pm 1.3$ & $1.2 \pm 0.3$ & $2.8 \pm 0.9$ \\
& & & & 0.3 & $1.0 \pm 0.8$ & $0.8 \pm 0.2$ & $1.7 \pm 0.8$ \\
\hline
\end{tabular}

Note. - References: 1. Portegies Zwart et al. (2010), 2. Rochau et al. (2010). The lower-mass limit is indicated as $m_{\text {min }}$.

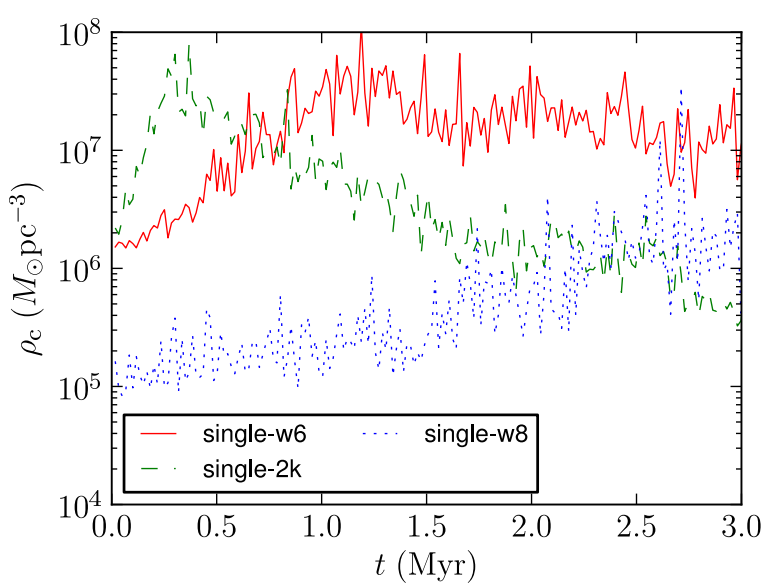

FIG. 4.- Time evolution of core density. These are averaged on all runs for models single-w6 and single-2k.

lund 1 is highly asymmetric, which could indicate that it had experienced mergers. Unfortunately, there is no data of runaway stars around this cluster, and little data is available about its internal kinematics. However, the effective cumulative radius:

$$
r_{\mathrm{eff}}(m)=\sqrt{\frac{\sum_{m_{i}>m}^{i} r_{i}^{2}}{i}},
$$

has been observed (Gennaro et al. 2011). Here, $r_{i}$ is the distance from the cluster center in projection for $i$-th massive stars with $m_{i}$. This is the geometric-averaged distance from the cluster center for stars with mass $m_{i}>m$. We calculated $r_{\text {eff }}(m)$ for our simulations in order to compare it with the observation. We took into account stars in a radius of $3 \mathrm{pc}$ to remove the effect of runaway stars. This radius is the same as the observed region, $\sim 2.3 \mathrm{pc}$. The results are shown in Figure 9. The smaller effective radius for more massive stars indicates mass segregation. With simulation $\mathrm{m} 2 \mathrm{k} 8 \mathrm{r} 3$, which has a density profile which is consistent to the observed cluster R136, we quantify the observation to $r_{\text {eff }} \sim 0.8 \mathrm{pc}$ at the minimum radius at massive end $\left(\sim 20 M_{\odot}\right)$ and $r_{\text {eff }} \sim 1.5$ pc for all stars down to $2.5 M_{\odot}$ (see also Figure 11 of Gennaro et al. 2011). The effective radius for stars with $\gtrsim 30 M_{\odot}$ is larger than than that at around $20-30 M_{\odot}$ in both the simulation and the observation. In the simulation the larger radius comes from some off-center massive stars, which are scattered from the cluster center. In contrast to the merger models, the single model singlew8, which initially has properties similar to of that of the current Westerlund 1, has not have sufficient time to evolve dynamically. From these results, we consider that Westerlund 1 is also a young massive cluster which is forming via hierarchical merger. Since Westerlund 1 (3-6 Myr) is slightly older than R136 ( 3Myr) but is still undergoing mergers, we expect that the sub-clusters 
1.

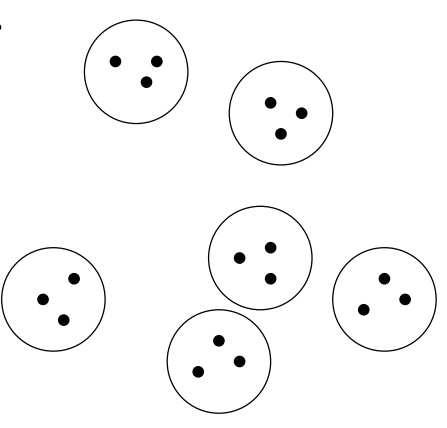

4.

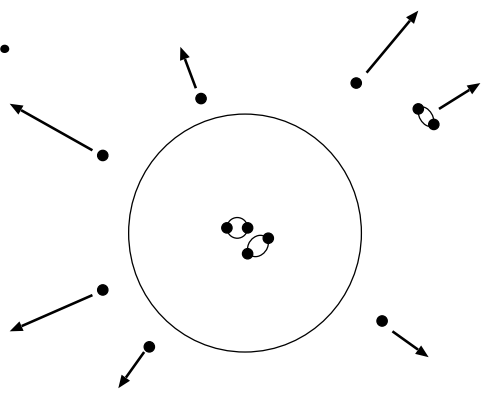

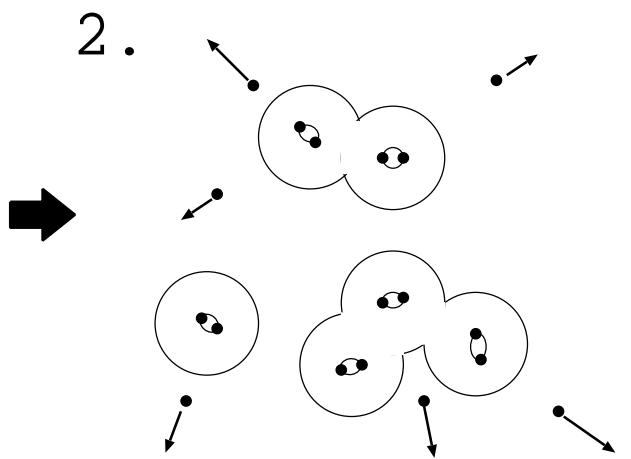

3 .

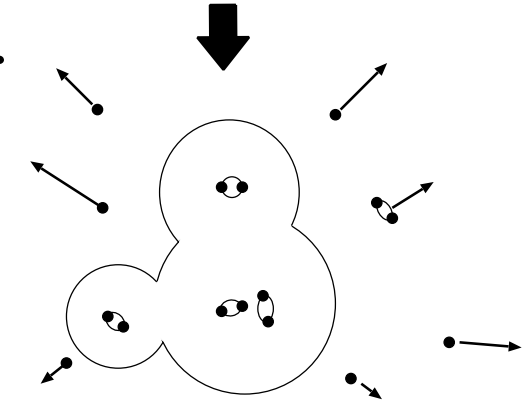

Fig. 5.- Formation of a star cluster through mergers. Sub-clusters form in a region of several pc (left top). They dynamically evolve and experience core collapse within $1 \mathrm{Myr}$. A BB form in each cluster and produce runaway stars by three-body scattering (right top). The sub-clusters merge hierarchically and the hardest BBs break or kick out the other BBs (bottom).
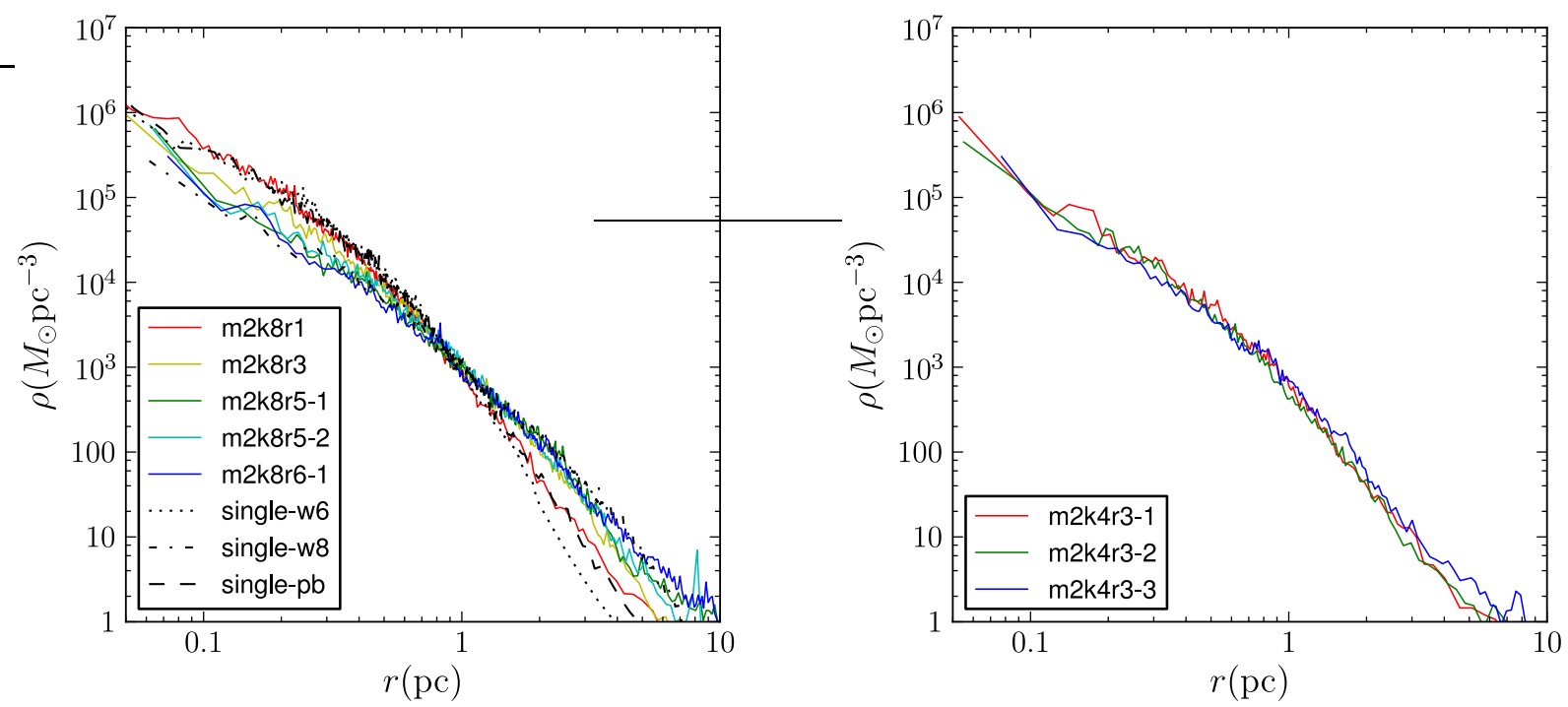

FiG. 6.- Density profiles of R136 (8-merger and single) models at $3 \mathrm{Myr}$ (left) and NGC 3603 (4-merger) models at 2.5 Myr (right).

of Westerlund 1 were born in a larger region than those of R136. We expect that based on the simulation, the numbers of runaway stars of Westerlund 1 are $7.0 \pm 2.5$ $(4.2 \pm 1.5), 6.8 \pm 3.2(4.1 \pm 1.8)$, and $11 \pm 6(6.8 \pm 3.7)$ for early-B, late-O, and early-O/WR stars assuming a Salpeter IMF with $1-100 M_{\odot}\left(0.3-100 M_{\odot}\right)$, respectively (see also table 3).

\section{DISTRIBUTION OF COLLIDED STARS}

Recent observations have identified several very massive stars with an estimated zero-age main-sequence mass of $\gtrsim 150 M_{\odot}$ in R136 and NGC 3603 (Crowther et al. 2010). Because collisions between stars easily happen in a dense cluster (Portegies Zwart et al. 1999), such very massive stars are a natural by-product of the dynamical evolution of a dense star cluster.

In Fig.10 we present the radial distribution and the mass distribution of stars that experienced collisions in our simulations. We adopted for adopting the maximum 

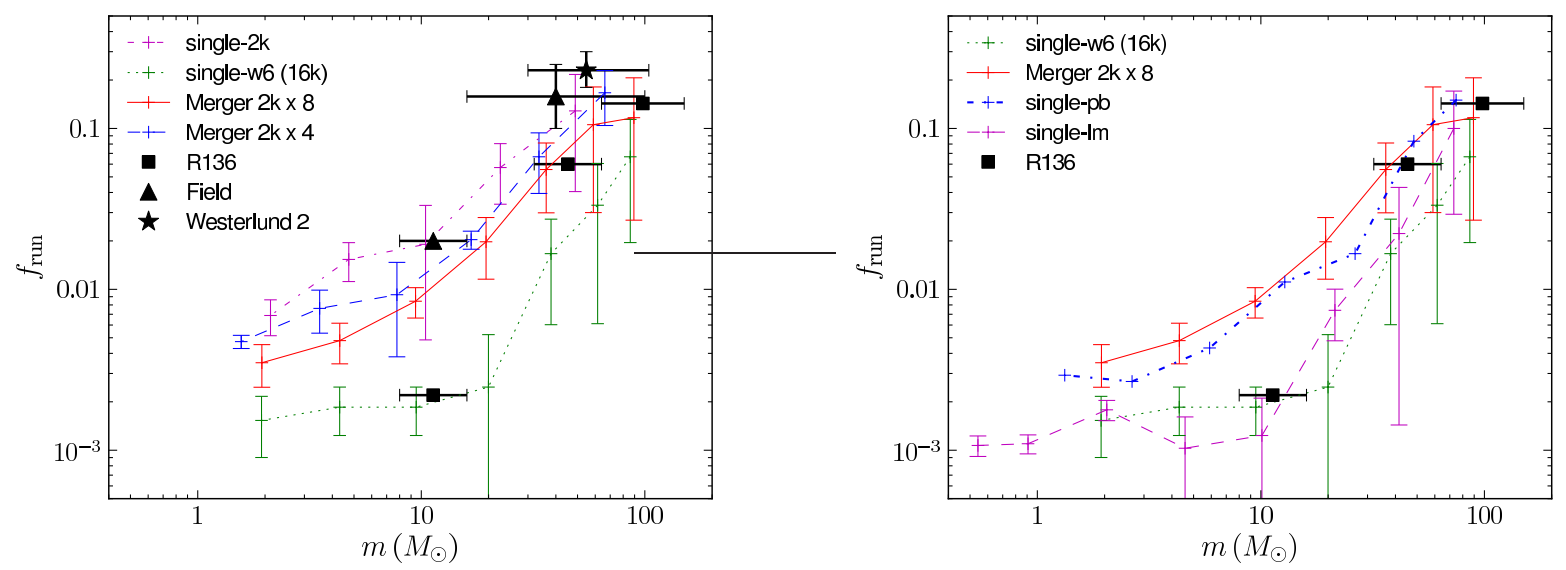

FIG. 7.- Fraction of runaway stars $(>30 \mathrm{~km} / \mathrm{s})$ to whole cluster as a function of mass at 3 Myr. Color curves show the results of our simulations. Left and right panels show comparisons between single and merger models and among single models, respectively. Square and triangle symbols show the runaway fraction of R136 and the field (from FPZ2011; Gvaramadze et al. 2010; Bestenlehner et al. 2011; Andersen et al. 2009; Zinnecker \& Yorke 2007). Star shows a fraction of isolated WR and early O-stars around Westerlund 2 (Tsuijmoto et al. 2007; Rauw et al. 2007; Nazé et al. 2008; Roman-Lopes et al. 2011).

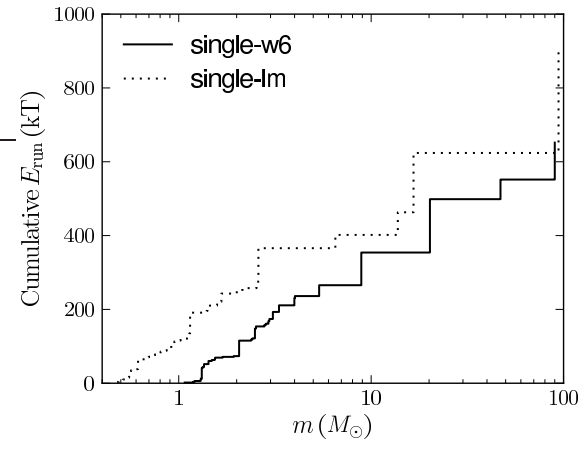

FIG. 8.- Kinetic energy of runaway stars as a cumulative function of mass. The energy is normalized by kT of each cluster. Note that the value of $\mathrm{kT}$ for model single-w6 is twice as large as that of single-lm because of the difference of their mean masses.

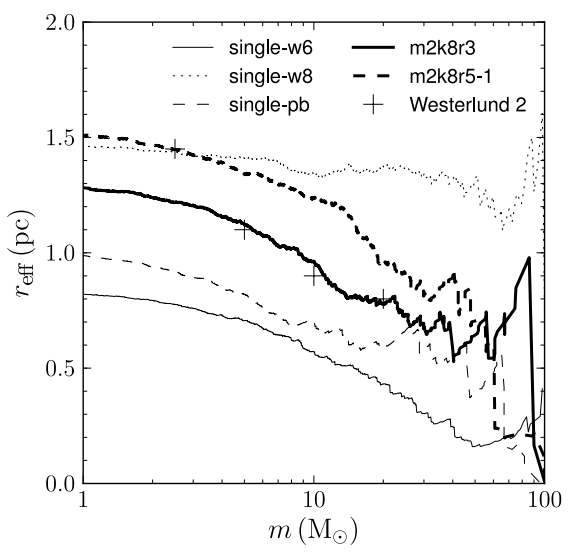

FIG. 9.- Effective cumulative radius for stars in $3 \mathrm{pc}$ (in projection) at $3.5 \mathrm{Myr}$. Data points of Westerlund 2 are from Gennaro et al. (2011). masses a star reaches during its lifetime rather than the mass at the end of the simulation, because the latter strongly depends on the mass-loss rate. For comparison, we also indicate the very massive stars observed in R136 and NGC 3603 (Crowther et al. 2010; Bestenlehner et al. 2011). In the left panel of Figure 10, we can see the effect of the mass segregation within $\sim 1 \mathrm{pc}$, while we find stars escaping at distances greater than 10 pc. The escaping massive stars formed in the merger models with $r_{\max }=$ 5-6 pc. The maximum mass that was produced in these simulations was around $240 M_{\odot}$, and three of these stars are ejected from the cluster as single runaways. Their velocities are similar, $\sim 60 \mathrm{~km} / \mathrm{s}$ in three dimensions, which corresponds to $\sim 35 \mathrm{~km} / \mathrm{s}$ in one dimension. This mass and velocity are very similar to those of VFTS 682 , which is located at $\sim 30 \mathrm{pc}$ from R136 in projection and seems to be escaping from R136 with one-dimensional velocity of $\sim 30 \mathrm{~km} / \mathrm{s}$ (Bestenlehner et al. 2011). With a current mass of VFTS 682 is $\sim 150 M_{\odot}$ its initial mass would $\lesssim 210 M_{\odot}$ (Bestenlehner et al. 2011).

Such very massive stars have been found also inside of R136. There are four massive stars with initial masses of $165-320 M_{\odot}$ in R136 (Crowther et al. 2010). Three of four massive stars in R136 are located in the inner most $0.1 \mathrm{pc}$ and the other one is $\sim 1 \mathrm{pc}$ (in projection) from the cluster center. In our simulations, we found 3-4 very massive stars in the cluster center in the case of merged clusters with $r_{\max }=5-6 \mathrm{pc}$. Model m2k8r6-1 evolves towards a distribution very similar to R136. In Figure 11. we present the radial distribution of merger products with $>100 M_{\odot}$.

We compared the distribution of massive stars in NGC 3603 with the simulations. There are four very massive stars with initial masses of $105-170 M_{\odot}$ in $\mathrm{NGC}$ 3603 , and two of them (A1a and A1b) form a binary (Crowther et al. 2010). We found 2-3 collided stars which exceed $100 M_{\odot}$ in each run. Some of the stellar merger products form binaries. We find a massive binary composed of stars that experienced a collision in model m2k4r3-3 and one composed of a collided star and $\mathrm{a} \sim 100 M_{\odot}$ normal star in model m2k4r3-1. We 
speculate, based on our simulation results, that massive $\left(>100 M_{\odot}\right)$ star could have been ejected from NGC 3603 (see right panels of figures 10] and 11), and could still roam the LMC.

The merger simulations that started with a relatively large $r_{\max }$ have mass and density profiles that are consistent with the observations, but the single clusters and merger simulations with small $r_{\max }$ are unable to reproduce the observations. In the latter cases, one very massive star $\gtrsim 400 M_{\odot}$ forms in the cluster as a result of runaway collisions (see Figure 10). However, there is no observational evidence of such a massive star in R136 or NGC 3603.

In the simulations with primordial binaries (singlepb), no massive runaway star which experienced collisions formed, whereas Banerjee et al. (2012) found some collided runaway stars in their $N$-body simulation models of R136 for a single cluster with primordial binaries. In our simulation, most of the massive stars collided into the most massive star via runaway collisions as is the case without primordial binaries, which is consistent with Portegies Zwart et al. (1999). We argue that the difference between our results and those presented in Baneriee et al. (2012) originate from the initial conditions. The major differences are the orbital period distribution of primordial binaries and their assumption that all binaries are born in the cluster core. We adopted the same binding energy for all binaries (orbital periods for massive binaries are $\sim 3 \times 10^{3}-10^{6}$ days) and distribute them in the same way in the cluster as the single stars. On the other hand, Banerjee et al. (2012) chose shorter periods of $0.5<\log _{10}(P /$ days $)<4$. They employed initial mass segregation; they initialized the heaviest stars in the cluster core. The combination of relatively tight binaries and a high degree of mass segregation stimulate strong dynamical encounters which may in their case have resulted in the stimulated formation of a massive runaway in the simulations with primordial binaries.

In Figure 11 we show that about half the stars that experienced one or more collisions are accompanied by another star. In simulations with merging clusters, one $\mathrm{BB}$ forms in each sub-cluster before it merges. The BBs in the sub-cluster centers participate in colliding encounters due to which they become more massive. After the sub-clusters merge, one of the BBs can be dynamically scattered and escapes from the cluster. We discuss binaries in section ??.

Stars that experience a collision are candidates for rapid rotation (Fryer \& Heger 2005). Several rapidly rotating massive stars have been found in recent observations, and possibly this rotation is the result of the earlier collision history of the star. The star VFTS 682, has a rotation velocity $>200 \mathrm{~km} / \mathrm{s}$ (Bestenlehner et al. 2011), and the $>20 M_{\odot}$ VFTS 102 , which is a runaway with $\sim 40 \mathrm{~km} / \mathrm{s}$ located in the 30 Doradus region, is also a rapid rotator $(>500 \mathrm{~km} / \mathrm{s}$ ) (Dufton et al. 2011). The rapid rotation of these stars could be explained by an earlier episode of mass transfer in a binary system or by collision with another star. Either of these probably occurred in the cluster center, from which it can easily have been ejected. In our simulations we find several rotating stars inside the model that reproduces the observations of R136 and a few are ejected as runaways (see figures
10] and 11).

\section{DISTRIBUTION OF BINARIES}

In Figure 12 we show the radial distribution of hard binaries (the binding energy is more than 1kT) at 3 Myr. In the simulations with primordial binaries, we find 306 such binaries at an age of 3 Myr, which is $\sim 60 \%$ of the primordial binary population. The majority of these binaries are primordial.

In the case of a single cluster with/without primordial binaries and merged clusters with $r_{\max }=1-3 \mathrm{pc}$, there is generally one $\mathrm{BB}$ in the cluster center. In the case of a merged cluster with $r_{\max }=5-6 \mathrm{pc}$, however, we found 3-7 BBs. Furthermore, we found two escaping binaries in model m2k8r6-1. They are located at 38 and $27 \mathrm{pc}$ from the cluster center and their velocities are $24 \mathrm{~km} / \mathrm{s}$ and $16 \mathrm{~km} / \mathrm{s}$, which are slightly slower than the velocity to be defined as runaway stars, but these are unbound. We also found off-center binaries in two of our merger models (models m2k8r5-1 and m2k8r6-2). They are located at 36 and $8 \mathrm{pc}$ from the cluster center, but their velocity is $\sim 10 \mathrm{~km} / \mathrm{s}$ and they are bound to the cluster. These binaries are remnants of the BBs that formed in sub-clusters and were kicked out after their host clusters merged. Therefore, they are very massive with a maximum mass $\sim 240 M_{\odot}$. Figure 13 shows the orbital periods and the total current masses of the binaries obtained from the simulations. The orbital period of the massive binaries located farther than 10 pc (marked by circle in the figure) is $\sim 500$ days. R145, which is a massive binary $\left(300+125 M_{\odot}\right)$ located at $\sim 20$ pc in projection from R136 and of which orbital period is 158.8 days (Schnurr et al. 2009; Fitzpatrick \& Savage 1984), has similar characteristics to these escaping binaries.

\section{SUMMARY}

We performed simulations of merging and single star clusters and compared the radial distribution of massive stars and the mass distribution of runaway stars to observations. We found that clusters that assembled from the hierarchical merging of multiple sub-clusters can explain the characteristics (mass distribution, mass segregation, runaway stars, massive stars, and massive binaries) of young dense clusters such as R136 in the LMC and NGC 3603, Westerlund 1, and 2 in the Milky Way.

We have set-up our simulations in such a way that the sub-clusters have a sufficiently short relaxation time that they experience core collapse before they merge and as a consequence the large merged cluster is in a state of core collapse when it forms. This results in the mass segregation of the merger remnants. Each sub-cluster experiences core collapse which leads to a collision runaway and the consequent production of a very massive $\left(>100 M_{\odot}\right)$ star in the process. After the clusters have merged the BBs gather in the center of the merged cluster. The majority of them are ionized or ejected from the cluster out by binary-binary, in fact BB-BB, interactions. These processes lead to the formation of very massive stars, consistent with those observed in R136 and NGC 3603, massive runaway stars such as VFTS 682, and massive binaries in the periphery of young cluster like R145. The post-core-collapse evolution of the sub-clusters drives the expansion of the core by the ejection of a large number 

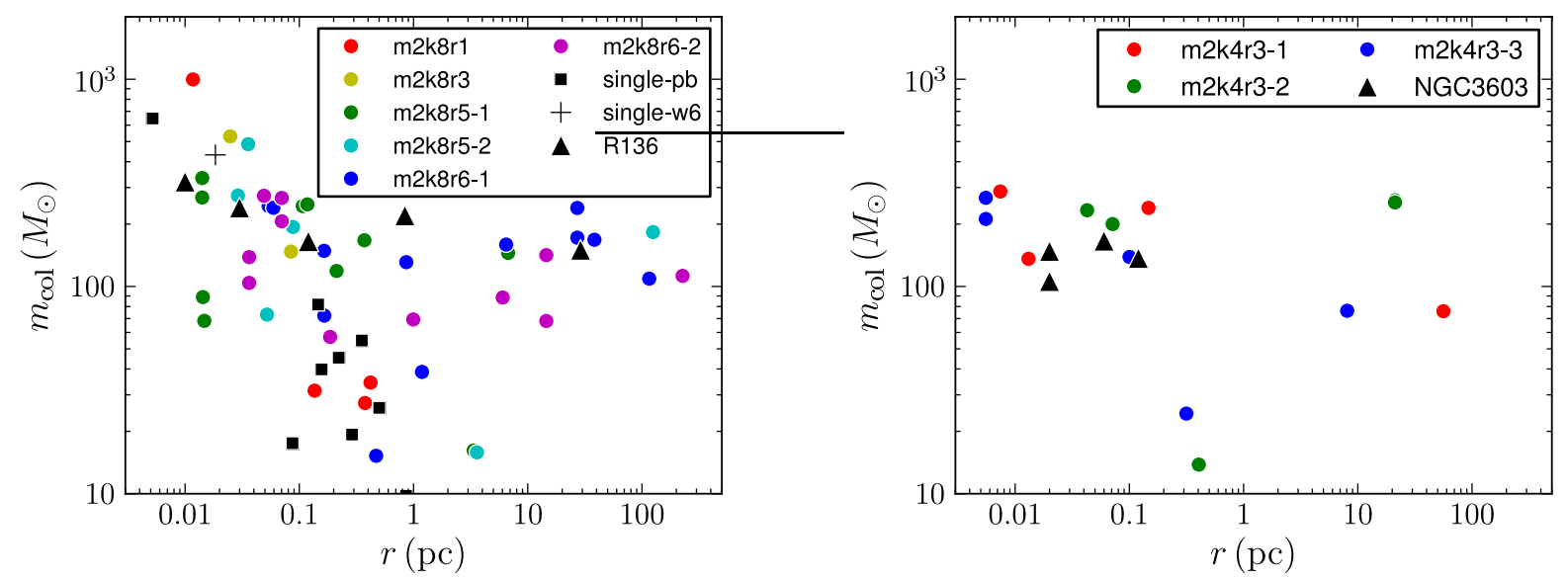

FIG. 10.- Radial and mass distribution of collided stars at $3 \mathrm{Myr}$ for 8-merger models (left) and 2.5 Myr for 4-merger models (right). Circles show merged cluster cases, whereas squares show a single case with primordial binaries. The cross shows the averaged value of single cluster models without primordial binaries (single-w6). In the case of single clusters, only one runaway collision star grows in a cluster. Black triangles show very massive stars $\left(>100 M_{\odot}\right)$ found in R136 and NGC 3603 (Crowther et al. 2010; Bestenlehner et al. 2011). The right top green point in the right panel is a binary which have very similar masses.
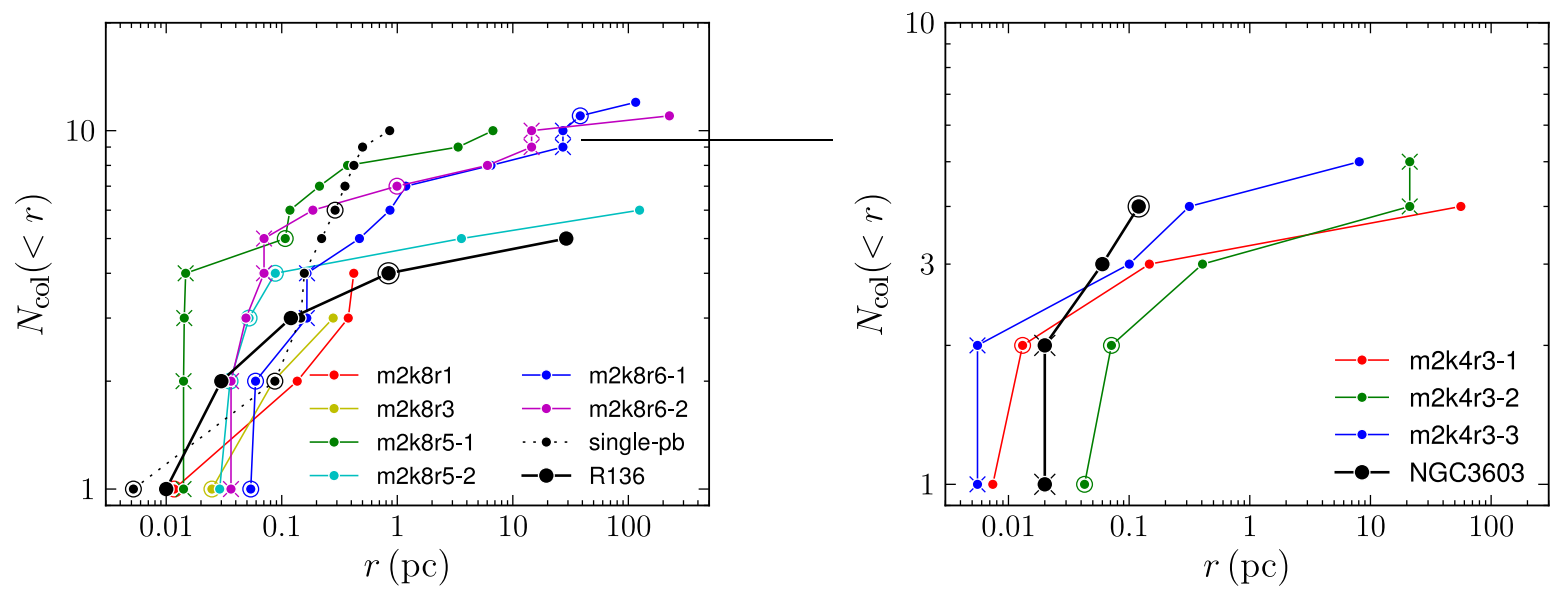

FIG. 11. - Cumulative radial distribution of stars which experienced collisions and with $>100 M_{\odot}$ at 3 Myr for R136 models (left) and $2.5 \mathrm{Myr}$ for NGC 3603 models (right). Crosses indicate binaries whose companions are also collided stars (two crosses at the same radius are a pair). Surrounded circles indicate binaries whose companions are not collided stars.

of runaway stars. The distribution of massive stars and the core density of the star clusters which formed via hierarchical mergers are consistent with those of R136 and NGC 3603.

The initial conditions of our simulations are constructed such that core collapse occurs at an age of $\sim 0.3$ Myr. This fine tuning is required in order to explain the coexistence of a rich population of runaways, the presence of a BB in the cluster center (or outside) and the relatively low density of the observed clusters. Observing a star cluster in a state of cluster collapse at an age $\lesssim 1$ Myr would provide a confirmation for formation model for massive clusters and the co-production of runaway stars and extremely massive single stars.

If we adopt single clusters with initial conditions for which the distribution of the massive stars is consistent with observations, our simulated clusters are insufficiently dense to experience core collapse within a few Myr and to form runaway stars within the age of the observed clusters. On the other hand, initial single clusters that are sufficiently dense to experience core collapse within $3 \mathrm{Myr}$ can produce a sufficiently large number of runaways compared to the observed number found around R136, but they are considerably more concentrated than the observed clusters. Single clusters with primordial binaries mediates the number of runaway stars, but also lead to a too concentrated density profile at later time.

Westerlund 1 and 2 are also candidates of merged clusters, in particular because they appear to be rather clumpy and they are strongly mass segregated (Gennaro et al. 2011; Ascenso et al. 2007). The degree of mass segregation in our merger simulations is consistent with the observed cumulative effective radius of Westerlund 1 . The fraction of runaway stars in the vicinity of Westerlund 2 is consistent with the result of our simulations in which multiple clusters merge to one.

Our results support the claim that some young dense clusters, such as R136, NGC 3603, Westerlund 1, and 2, 


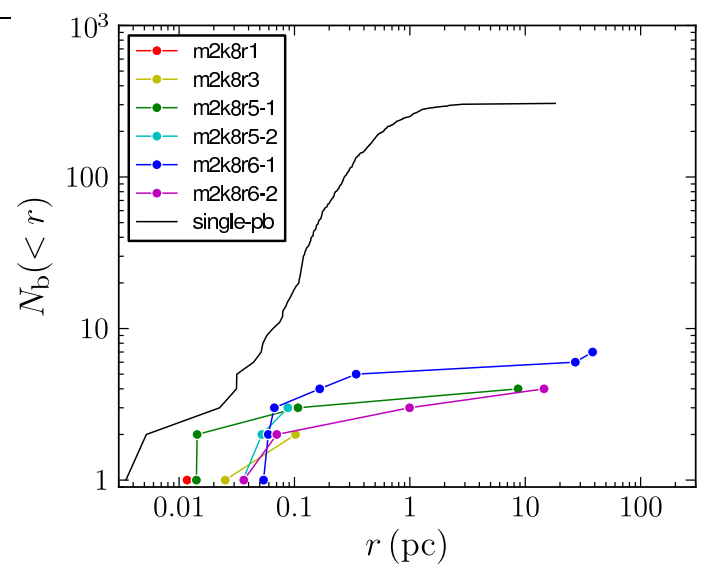

FIG. 12.- Cumulative radial distribution of hard binaries at 3 Myr. Colored points show merger models, and the black curve shows a single model with primordial binaries.

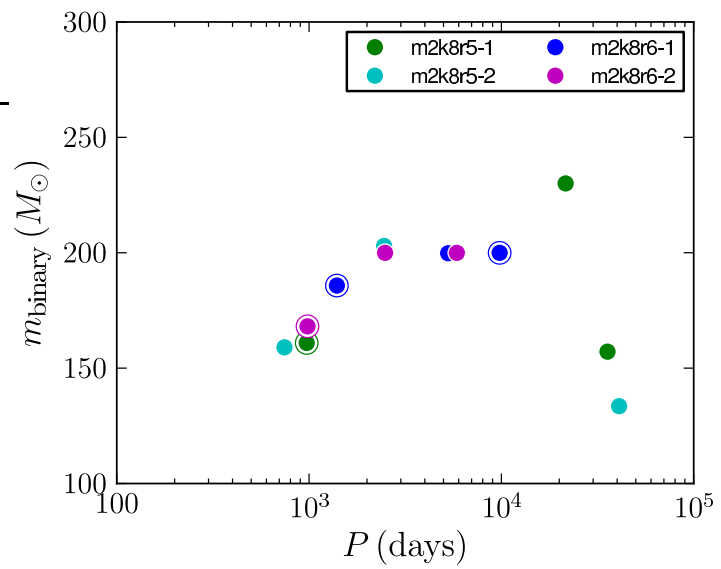

FIG. 13.- Orbital periods and total masses of binaries at $3 \mathrm{Myr}$. Points with circles indicate binaries which are located at $>5 \mathrm{pc}$ from the cluster center. formed via mergers of smaller clusters. We further argue that the small sub-clusters which are best suited to reproduce the observed characteristics of the young dense clusters are also the building blocks for star clusters elsewhere in the Milky Way, and are therefore responsible for the production of $\mathrm{O}$ and $\mathrm{B}$ runaway stars in the Galactic disk. The typical scale of the cluster is $M \sim 6000 M_{\odot}$, and the fraction of runaway stars among O-type stars is about 10-20\%, and drops to a few percent for B-type stars (FPZ2011). Clusters more massive than those studied here may also have formed through the merging of multiple smaller sub-clusters. We argue that the fundamental building-block cluster with which we are able to explain the density profile of observed star clusters and at the same time the rich population of massive early B and $\mathrm{O}$ stars in the vicinity has a mass of $\lesssim 10000 M_{\odot}$.

We thank Keigo Nitadori for developing the code, Masaki Iwasawa for fruitful discussions, and Alexander Rimoldi for careful reading. We also thank the anonymous referee for useful comments. This work was supported by Research Fellowship of the Japan Society for the Promotion of Science (JSPS) for Young Scientists, the Netherlands Research Council NWO (grants VICI [\#639.073.803], AMUSE [\#614.061.608] and LGM [\# 612.071.503]), the Netherlands Research School for Astronomy (NOVA), and HPCI Strategic Program Field 5 'The origin of matter and the universe'. Numerical computations were carried out on the Cray XT4 at the Center for Computational Astrophysics (CfCA) of the National Astronomical Observatory of Japan and the Little Green Machine at Leiden University.

\section{REFERENCES}

Aarseth, S. J. \& Hills, J. G. 1972, A\&A, 21, 255

Andersen, M., Zinnecker, H., Moneti, A., McCaughrean, M. J., Brandl, B., Brandner, W., Meylan, G., \& Hunter, D. 2009, ApJ, 707, 1347

Ascenso, J., Alves, J., Beletsky, Y., \& Lago, M. T. V. T. 2007, A\&A, 466, 137

Banerjee, S., Kroupa, P., \& Oh, S. 2012, ApJ, 746, 15

Bestenlehner, J. M., Vink, J. S., Gräfener, G., Najarro, F., Evans, C. J., Bastian, N., Bonanos, A. Z., Bressert, E., Crowther, P. A., Doran, E., Friedrich, K., Hénault-Brunet, V., Herrero, A., de Koter, A., Langer, N., Lennon, D. J., Maíz Apellániz, J., Sana, H., Soszynski, I., \& Taylor, W. D. 2011, A\&A, 530, L14+

Bonnell, I. A., Bate, M. R., \& Vine, S. G. 2003, MNRAS, 343, 413

Bonnell, I. A., Smith, R. J., Clark, P. C., \& Bate, M. R. 2011, MNRAS, 410, 2339

Bonnell, I. A., Vine, S. G., \& Bate, M. R. 2004, MNRAS, 349, 735

Brandl, B. R., Portegies Zwart, S. F., Moffat, A. F. J., \& Chernoff, D. F. 2007, in Astronomical Society of the Pacific Conference Series, Vol. 367, Massive Stars in Interactive Binaries, ed. N. St.-Louis \& A. F. J. Moffat, 629

Brüns, R. C. \& Kroupa, P. 2011, ApJ, 729, 69

Brüns, R. C., Kroupa, P., Fellhauer, M., Metz, M., \& Assmann, P. 2011, A\&A, 529, A138

Casertano, S. \& Hut, P. 1985, ApJ, 298, 80

Clark, J. S., Negueruela, I., Crowther, P. A., \& Goodwin, S. P. 2005, A\&A, 434, 949

Clarke, C. J. \& Pringle, J. E. 1992, MNRAS, 255, 423

Crowther, P. A. \& Dessart, L. 1998, MNRAS, 296, 622
Crowther, P. A., Schnurr, O., Hirschi, R., Yusof, N., Parker, R. J., Goodwin, S. P., \& Kassim, H. A. 2010, MNRAS, 408, 731

Dufton, P. L., Dunstall, P. R., Evans, C. J., Brott, I., Cantiello, M., de Koter, A., de Mink, S. E., Fraser, M., Hénault-Brunet, V., Howarth, I. D., Langer, N., Lennon, D. J., Markova, N., Sana, H., \& Taylor, W. D. 2011, ApJ, 743, L22

Evans, C. J., Walborn, N. R., Crowther, P. A., Hénault-Brunet, V., Massa, D., Taylor, W. D., Howarth, I. D., Sana, H., Lennon, D. J., \& van Loon, J. T. 2010, ApJ, 715, L74

Fitzpatrick, E. L. \& Savage, B. D. 1984, ApJ, 279, 578

Fryer, C. L. \& Heger, A. 2005, ApJ, 623, 302

Fujii, M., Iwasawa, M., Funato, Y., \& Makino, J. 2008, ApJ, 686, 1082

-. 2009, ApJ, 695, 1421

Fujii, M. S. \& Portegies Zwart, S. 2011, Science, 334, 1380

Gennaro, M., Brandner, W., Stolte, A., \& Henning, T. 2011, MNRAS, 412, 2469

Girichidis, P., Federrath, C., Banerjee, R., \& Klessen, R. S. 2011, MNRAS, 413, 2741

Gualandris, A., Portegies Zwart, S., \& Eggleton, P. P. 2004, MNRAS, 350, 615

Gürkan, M. A., Freitag, M., \& Rasio, F. A. 2004, ApJ, 604, 632

Gutermuth, R. A., Megeath, S. T., Myers, P. C., Allen, L. E.,

Pipher, J. L., \& Fazio, G. G. 2009, ApJS, 184, 18

Gvaramadze, V. V. \& Gualandris, A. 2011, MNRAS, 410, 304

Gvaramadze, V. V., Kroupa, P., \& Pflamm-Altenburg, J. 2010 A\&A, 519, A33+

Harayama, Y., Eisenhauer, F., \& Martins, F. 2008, ApJ, 675, 1319 
Hurley, J. R., Pols, O. R., \& Tout, C. A. 2000, MNRAS, 315, 543

King, I. R. 1966, AJ, 71, 64

Kruijssen, J. M. D., Maschberger, T., Moeckel, N., Clarke, C. J., Bastian, N., \& Bonnell, I. A. 2012, MNRAS, 419, 841

Lada, C. J. \& Lada, E. A. 2003, ARA\&A, 41, 57

Leonard, P. J. T. \& Duncan, M. J. 1988, AJ, 96, 222

Mackey, A. D. \& Gilmore, G. F. 2003, MNRAS, 338, 85

McMillan, S. L. W., Vesperini, E., \& Portegies Zwart, S. F. 2007, ApJ, 655, L45

Mengel, S. \& Tacconi-Garman, L. E. 2009, Ap\&SS, 324, 321

Moeckel, N. \& Bonnell, I. A. 2009, MNRAS, 400, 657

Nazé, Y., Rauw, G., \& Manfroid, J. 2008, A\&A, 483, 171

Nitadori, K. \& Makino, J. 2008, New Astronomy, 13, 498

Portegies Zwart, S. F., Makino, J., McMillan, S. L. W., \& Hut, P. 1999, A\&A, 348, 117

Portegies Zwart, S. F. \& McMillan, S. L. W. 2002, ApJ, 576, 899

Portegies Zwart, S. F., McMillan, S. L. W., \& Gieles, M. 2010, ARA\&A, 48, 431

Rauw, G., Manfroid, J., Gosset, E., Nazé, Y., Sana, H., De

Becker, M., Foellmi, C., \& Moffat, A. F. J. 2007, A\&A, 463, 981
Rochau, B., Brandner, W., Stolte, A., Gennaro, M., Gouliermis, D., Da Rio, N., Dzyurkevich, N., \& Henning, T. 2010, ApJ, 716, L90

Roman-Lopes, A., Barba, R. H., \& Morrell, N. I. 2011, MNRAS, 416,501

Saitoh, T. R., Daisaka, H., Kokubo, E., Makino, J., Okamoto, T., Tomisaka, K., Wada, K., \& Yoshida, N. 2011, in IAU Symposium, Vol. 270, IAU Symposium, ed. J. Alves, B. G. Elmegreen, J. M. Girart, \& V. Trimble, 483-486 Salpeter, E. E. 1955, ApJ, 121, 161

Sana, H. \& Evans, C. J. 2011, in IAU Symposium, Vol. 272, IAU Symposium, ed. C. Neiner, G. Wade, G. Meynet, \& G. Peters, $474-485$

Schnurr, O., Moffat, A. F. J., Villar-Sbaffi, A., St-Louis, N., \& Morrell, N. I. 2009, MNRAS, 395, 823

Tanikawa, A. \& Fukushige, T. 2009, PASJ, 61, 721

Tsujimoto, M., Feigelson, E. D., Townsley, L. K., Broos, P. S., Getman, K. V., Wang, J., Garmire, G. P., Baba, D., Nagayama T., Tamura, M., \& Churchwell, E. B. 2007, ApJ, 665, 719

Yu, J., de Grijs, R., \& Chen, L. 2011, ApJ, 732, 16

Zinnecker, H. \& Yorke, H. W. 2007, ARA\&A, 45, 481 